Journal of Engineering and Applied Sciences 15 (1): 67-73, 2020

ISSN: 1816-949X

(C) Medwell Journals, 2020

\title{
Cardiovascular Risk in Workers of the Area of Prevention and Emergency Control of the Oil Sector
}

\author{
${ }^{1}$ Irlena Ahumada, ${ }^{1}$ Jorge Posada, ${ }^{1}$ Ivan Orjuela, ${ }^{1}$ Jose Palacio, ${ }^{2}$ Carlos Vidal and ${ }^{1}$ Carlos Severiche \\ ${ }^{1}$ Corporacion Universitaria Minuto de Dios. Barranquilla, Atlantico, Colombia \\ ${ }^{2}$ Universidad De Santander. Valledupar, Cesar, Colombia
}

\begin{abstract}
The cardiovascular risk was estimated in workers in the area of prevention and control of emergencies of a company in the oil sector. It is a cross-sectional study of prevalence in the population aged 24-50 years, with a representative sample of 52 people without known cardiovascular disease, who were given a predesigned survey in order to assess the prevalence of cardiovascular risk factors, Total Cholesterol (CT), HDL-c, LDL-c, Triglycerides (TGs) and glucose were measured, values of blood pressure, abdominal perimeter, height and weight were taken. The habit of smoking was questioned. The most important cardiovascular risk factors and which represent a higher prevalence were dyslipidemia with $6 \%$ and obesity with $24 \%$. A low prevalence of cardiovascular risk factors was found, as well as the low probability of suffering a CVD at 10 years.
\end{abstract}

Keywords: Cardiovascular diseases, risk factors, prevalence, oil sector, occupational health

\section{INTRODUCTION}

Health is essential for the exercise of any activity, WHO defines it as "the state of complete physical, mental and social wellbeing and not only the absence of diseases or diseases (Balog, 2017), it means that the set of these three elements they produce a balance that would grant a person a well-being but there are other conditions that can alter or produce negative effects on an individual (Thimbleby, 2013).

For its part, taking into account that the disease is not something Foreign to the human condition but is part of its nature, in the work is exposed to a series of events that in one way or another can be harmful to man as employee (Wachter and Yorio, 2014).

When reviewing global and national statistics, it can be concluded that cardiovascular risk factors represent the link to the highest morbidity and mortality in men and women. The Cardiovascular Risk (CVR) is defined as the probability of presenting a cardiovascular disease in a determined period of time, generally from 5-10 years (Hajar, 2017). The cardiovascular risk factor corresponds to a biological or behavioral characteristic present in a healthy person that is independently related to the subsequent development of a cardiovascular disease that is it increases the probability of the presentation of said disease (Assimes and Roberts, 2016).

Cardiovascular Diseases (CVD) are the leading cause of death worldwide. Every year more people die from cardiovascular disease than from any other cause. Cardiovascular Diseases (CVD) are a public health problem worldwide due to their high prevalence (Pagidipati and Gaziano, 2013). According to the World Health Organization, the ECV is responsible for 32 coronary events and cerebrovascular accidents of which $40-70 \%$ are fatal in developed countries. It is estimated that this problem is much greater in developing countries and it is considered that millions of people suffer from risk factors, such as high blood pressure, smoking, diabetes, dyslipidemias and inadequate diet which are commonly poorly diagnosed (Shah and Afzal, 2013).

Most CVD can be prevented by acting on behavioral risk factors such as tobacco use, unhealthy diets and obesity, physical inactivity or harmful alcohol consumption, using strategies that cover the entire population. Of course, predisposing and genetic factors also play a role in hypertension, diabetes mellitus and disorders of lipid metabolism. Many of the risk factors facilitate the development of arteriosclerosis which is a significant pre-condition for the onset of coronary heart disease (Chan and Woo, 2010).

Cardiovascular risk factors include stress. It is defined as a set of physiological and psychological reactions that the organism experiences when subjected to strong demands (Soler and Ruiz, 2010). Stress is considered as the trigger of many cardiovascular diseases in susceptible organisms such as cerebral ischemia (stroke) and myocardium (chest angina, symptomatic or asymptomatic infarction). It is also associated with arterial hypertension and malignant arrhythmias (Balcıoglu and Muderrisoglu, 2015). 
The cardiovascular response to stress is associated with: increase in heart rate, blood pressure, cardiovascular tone and reactivity, free fatty acids, triglycerides, total cholesterol on the other hand decrease in HDL, variability of heart rate and insulin efficacy (Upadhyay, 2015).

Studies at a national level have revealed that, in Colombia, between 1998 and 2011, the main cause of mortality was due to diseases of the circulatory system. In 2006 it was revealed that the incidence is 133, represented in 1 of every 100,000 inhabitants. In 2011, a study was conducted in the municipality of Santa Rosa de Osos, Antioquia, in which the most important cardiovascular risk factors were low physical activity (56.6\%), central obesity (52.7\%), dyslipidemias (35.3\%), smoking (19.0\%), metabolic syndrome (19.6\%) and obesity by body mass index (17.4\%). The risk factors with the highest population attributable risk were arterial hypertension (17.1\%), total cholesterol/high density lipoprotein index (16.4\%) and low physical activity (15.7\%) (Patiño-Villada et al., 2011). During the aforementioned period, of the total of deaths, 323,037 were men, representing a crude average annual mortality rate of 108.9 per 100,000 . Likewise, studies reveal that dyslipidemia and sedentary life represent one of the main cardiovascular risk factors, where $31.4 \%$ present medium, moderate or high risk of suffering a heart attack in the following 10 years according to the Framminghan scale (Mensah et al., 2017). Worldwide, during 2012, 17.5 million people died, $17 \%$ of all deaths in the world, including 7.4 million due to coronary heart disease and 6.7 million to cardiovascular accidents (Ofori-Asenso and Garcia, 2016).

It is general knowledge that the human factor represents within any organization, the most important resource and, therefore, to achieve an excellent development of work activities, workers must be provided with an adequate working environment, with the order to preserve the health of these. Because of its complex etiology, only a very small proportion of cases of cardiovascular disease is recognized as occupational disease (Posada et al., 2019).

Many countries, however, admit that occupational exposure contributes to the development of CVD (sometimes defined as work-related diseases). Working conditions and job demands play an important role in the multifactorial process that causes these diseases, but differentiating the role of individual causal components is very difficult (Braveman and Gottlieb, 2014).

Daily activities and work in any sector influence the development of cardiovascular accidents and this, is closely related to physical condition, habits and lifestyles. In Spain, for example, one of the main causes of temporary disability in the workplace is attributed to CVD and is responsible for $45 \%$ of deaths in the present country (Mora-Pabón, 2018). Studies reveal that each worker has individual parameters related to the type of work activity developed that can influence the development, presentation and impact on cardiovascular risk factors (Gómez, 2016). Conditions such as dyslipidemia is also one of the most relevant factors due to its prevalence (52\%) in relatively young populations, followed by smoking (35\%) and obesity (22\%) (Yusufali et al., 2015).

In Brazil, studies revealed that in the year 1970 coronary heart disease represented the main cause of death. During the last decades due to the development of daily life, the change of eating habits and physical activities, there was an increase in the proportions of overweight, evidencing in the younger sector resulting in the appearance of diabetes and coronary diseases (Bhurosy and Jeewon, 2014).

Among CVD, hypertension has the highest annual rate of new cases in workers aged 35-64 years. New cases appear in approximately $1 \%$ of this population each year. The following frequency disorders are coronary heart disease (8-92) new cases of acute heart attack per 10,000 males per year) (Sanchis-Gomar et al., 2016).

\section{MATERIALS AND METHODS}

Type of study: For the study, the methodology that was proposed was cross-sectional descriptive type, since from surveys and medical evaluations were determined data of the workers of the area of prevention and control of emergencies of oil company.

Population and sample: For this project, the area of prevention and control of emergencies of oil company integrated by 52 workers, male.

\section{Inclusion criteria:}

- Workers belonging to the emergency care and prevention area who were between 30 and 74 years old were included

- No history of known coronary disease

- $\quad$ Desire to participate in the study

\section{Exclusion criteria:}

- $\quad$ Female sex

Methodological instrument: Studies performed show different techniques that have been used for the identification of cardiovascular risks. The Framingham heart study model is one of the most used to measure levels of cardiovascular risk, for example, calculating the absolute risk of an asymptomatic individual. You can determine if you will need immediate intervention with a comprehensive approach (Mendis, 2005). 
The Frammingham Model uses a scoring method based on the following variables: age, sex, HDL-cholesterol, total cholesterol, systolic blood pressure, smoking (Yes-No), diabetes (Yes-No) and left ventricular hypertrophy. With the above data it is possible to calculate the coronary risk at 10 years, which includes stable angina, myocardial infarction and coronary death (Bitton and Gaziano, 2010).

It is good to point out that this tool for risk assessment has limitations, since, it has been developed in the population of Framingham (a small North American town near the city of Boston) and must be adjusted when applied to populations with a lower incidence of cardiovascular events. This fact is of fundamental importance, since many interventions are recommended after the individual exceeds a certain threshold of risk of suffering 10 years events (fifteen).

It constitutes a basic pillar and in different ways it is widely used for therapeutic decision making based on the risk estimation provided by the method when introducing the risk characteristics of the specific patient (Juhnke et al., 2016).

For the present study, the classic Framingham risk tables and by categories (Wilson) were taken as reference. Where the method was performed by scoring based on the following variables:

- Age

- HDL cholesterol

- Total cholesterol

- $\quad$ Blood pressure

- $\quad$ Smoking

- Diabetes

The above information was collected based on Sociodemographic surveys, being the main source for calculating coronary risk at 10 years.

Statistical data analysis: For the treatment of the data, collected after the application of the Sociodemographic and Labor Surveys to the workers included in our sample. The tabulation of the information was carried out, Microsoft Excel being our tool for this purpose. It was verified that the data complied with a normal distribution, to verify that there was no bias in the information. Included, the measures of central tendency (fashion, medium, medium), frequency distribution, typical errors, etc. were determined.

In order to reduce information biases, a preliminary meeting was held among the interviewers to discuss the handling of data collection instruments (Surveys) in order to achieve their correct completion in a standardized manner.

In addition, quality control of the processed formats was carried out during the field work, in case of erroneous completion of the surveys; the interviewer would return the format that presented inconsistencies or missing data. The objectives of the study and each one of the procedures that would be carried out were explained to the workers in the area of prevention and control of emergencies and the confidentiality of the data provided by them was guaranteed, with the informed consent given to them.

A pilot test was conducted in order to know the difficulties that may arise during the application of the instrument and the average time of completion of the same.

Ethical considerations: The protection of the privacy of persons was guaranteed, according to the Declaration of Helsinki of 200867 and the provisions of resolution 08430 of 1993 of the Ministry of Health of the Republic of Colombia, 68 on informed consent and investigations with living beings. All the people studied were informed about the objectives of the study and the exclusive use of the data for scientific purposes. The results of the research were returned to the participants and to the cooperating institution.

Operation of variables: Surveys were conducted in which the following variables were recorded: Age, sex, smoking, alcoholism, diabetes, level of education (baccalaureate, technical, technological, university, specialization, masters), civil status, seniority in the company, position held.

The person who received treatment or knew by medical diagnosis was considered as diabetic. Likewise, blood pressure was taken and recorded according to the JNC 8 and weight and height were determined for the calculation of BMI.

\section{RESULTS AND DISCUSSION}

Table 1 shows the sociodemographic characteristics of the population studied. Next, in Table 2 we observe the prevalence of smoking habit according to age. Regarding the position, it was found that the highest percentage of consumption was presented in the operators with $69 \%$ and workers with technological level of education reported a tobacco consumption of $69 \%$.

Table 3 shows a prevalence of workers with overweight of $56 \%$, included in the age range of 30-39 years with $36 \%$. It was determined, in turn, that all workers who present with dyslipidemia are overweight.

The cardiovascular risk factor, according to age in relation to the presence of hypertension, showed that $67 \%$ of workers surveyed presented high blood pressure levels compatible with arterial hypertension in the age range of 38-42 years and 33\% in ages from 34-38 years. In this same relation, based on the existence of pre-hypertension, it was evident that the highest percentage of pre-hypertensive patients are between 42 and 46 years, representing $55 \%$ of the total of the respondents. On the other hand, at the ages of 30-34 years, no patients with this condition were found. 
J. Eng. Applied Sci., 15 (1): 67-73, 2020

Table 1: Sociodemographic characteristics

\begin{tabular}{ll}
\hline Variables & $\mathrm{N}(50)$ \\
\hline Age group & \\
$30-34$ & 14 \\
$34-38$ & 10 \\
$38-42$ & 11 \\
$42-46$ & 9 \\
$46-50$ & 5 \\
$50-56$ & 1 \\
Position & \\
Supervisor & 6 \\
Tablers & 6 \\
Operator & 32 \\
Staff & 6 \\
Civil status & \\
Single & 10 \\
Married & 27 \\
Separated & 1 \\
Free union & 12 \\
Seniority in the company (years) & \\
Less than a & 4 \\
From 1-5 & 15 \\
From 5-10 & 23 \\
From 10-15 & 6 \\
More than 15 & 28 \\
Level of education & Academic \\
Technological &
\end{tabular}

Table 2: Prevalence of smoking

\begin{tabular}{ll}
\hline Variables & N(50) \\
\hline Smoking & \\
Smokers & 26 \\
No smoking & 74 \\
Age & \\
30 & 15 \\
33 & 15 \\
34 & 8 \\
36 & 8 \\
37 & 15 \\
38 & 8 \\
41 & 8 \\
42 & 8 \\
43 & 8 \\
46 & 8 \\
Level of education & \\
Academic & 31 \\
Technological & 69 \\
Position & \\
Operator & 69 \\
Supervisor & 15 \\
Console technician & 8 \\
Staff & 8 \\
\hline
\end{tabular}

Table 3: Body mass index

\begin{tabular}{lc}
\hline Variable & $\mathrm{N}(50)$ \\
\hline IMC & 56 \\
Overweight & 24 \\
Obese & \\
Age with overweight & 36 \\
$30-39$ & 20 \\
$40-49$ & 0 \\
$50-59$ & \\
Obese age & 10 \\
$30-39$ & 14 \\
$40-49$ & 0 \\
$50-59$ &
\end{tabular}

Table 4: Blood pressure

Variables $\quad \mathrm{N}(50)$

Blood pressure

Normal

\begin{tabular}{lr} 
Pre-hypertension & 18 \\
\hline
\end{tabular}

Hypertensive 4

Table 5: Pre-hypertension

Variables Percentage

Blood pressure

30-34

$34-38$

$38-42$

$42-46$

$46-50$

$\underline{50-56}$

Table 6: Hypertension

Variables Percentage

Blood pressure

34-38

33

38-42

Table 7: Mixed dyslipidemia

Variables

Mixed dyslipidemia

Age with mixed dyslipidemia

30-34

Table 8: Prevalence of diabetes mellitus

Variables $\mathrm{N}(50)$

Glucose values (mg dL $\left.\mathbf{~ L}^{-1}\right)$

36-58

58-80

80-102

102-124

124-146

146-168

$\underline{168-190}$

$\begin{array}{r}4 \\ 24 \\ 64 \\ 4 \\ 2 \\ 0 \\ 2 \\ \hline\end{array}$

In general terms, it was found that, of the total workers surveyed, $72 \%$ had normal blood pressure, 22\% had pre-hypertension and 6\% had high blood pressure in the range of arterial hypertension without a previous diagnosis of the same (Table 4-6).

When assessing the risk factor of mixed dyslipidemia, it was found that three of the workers surveyed had this pathology in the ages between 30 and 34 years, where one of them presented as a personal antecedent this condition and the other two were diagnosed during the study, representing $6 \%$ of the respondents. In Table 7, we have that of $100 \%$ of the population, 32\% had high cholesterol, 40\% hypertriglyceridemia and 10\% HDL levels below the normal range, with a higher age index of 30-39 years.

Table 8 shows the assessment of diabetes mellitus, based on Fasting Glucose, it was found that the highest proportion of surveyed workers are between $80-102 \mathrm{mg}$ $\mathrm{dL}^{-1}$ with 64 and $2 \%$ represent the values higher (168-190 $\mathrm{mg} \mathrm{dL}^{-1}$ ) glucose. Among the respondents, a worker with a personal history of diabetes mellitus was found. 
Table 9: Abdominal circumference

\begin{tabular}{lc}
\hline Variable & $\mathrm{N}(50)$ \\
\hline Abdominal circumference & \\
$80-86$ & 14 \\
$86-92$ & 6 \\
$92-98$ & 12 \\
$98-104$ & 40 \\
$104-110$ & 14 \\
$110-116$ & 8 \\
$116-122$ & 6 \\
\hline
\end{tabular}

Table 10: Global Framingham score and risk of CVD at 10 years

\begin{tabular}{lr}
\hline Workers & Risk \\
\hline$<2$ & 10 \\
2 & 8 \\
3 & 4 \\
4 & 2 \\
5 & 3 \\
6 & 3 \\
7 & 2 \\
8 & 3 \\
9 & 1 \\
10 & 1 \\
\hline Estimated risk: RCV Blow; RCV Moderadte 3; RCV High 3; Percentage
\end{tabular}
95

Table 11: Global Framingham score and 10 year CVD risk

\begin{tabular}{lc}
\hline Workers (\%) & Risk \\
\hline 0 & 4 \\
2 & 12 \\
3 & 12 \\
4 & 6 \\
5 & 3 \\
6 & 3 \\
7 & 0 \\
8 & 5 \\
9 & 3 \\
10 & 0 \\
16 & 3 \\
\end{tabular}

Regarding the abdominal perimeter as a basis for central obesity, it was found that $40 \%$ had abdominal circumference between 98 and $104 \mathrm{~cm}$ and 14\% of $104 \mathrm{~cm}$ and more, starting from the normal value for this up to $102 \mathrm{~cm}$ (Table 9).

When evaluating the global cardiovascular risk, 95\% of the respondents were classified as low risk and the remaining $6 \%$ between medium and high, distributed in $3 \%$ medium and $3 \%$ high (Table 10 ), while with the application of the method Framingham to estimate the risk of cardiovascular disease at 10 years was determined that only 4 of the workers presented a $0 \%$ risk at 10 years (Table 11). It should be noted that several surveys were excluded from the application of the surveys. The first group of excluded workers were those that when totaling the score the result was 1 and this value is not included in the stratification of the table, this group was a total of 7 workers. On the other hand, of the 50 participants, 4 of the workers were excluded because their total cholesterol levels were not within the parameters standardized by the Framingham method and therefore, could not be categorized. Conditions such as dyslipidemia were prevalent at the ages of 30-34 years, although, it accounted for $6 \%$ of the total population, coinciding with studies conducted in Spain, where dyslipidemia was also one of the most relevant factors due to its prevalence in the relatively young populations (Mohammadbeigi et al., 2015). On the other hand, for this study conducted in an oil company, the prevalence of smoking was $26 \%$ and the difference in percentages was not significant compared to Spanish studies (smoking $35 \%)$. For our study, a prevalent cardiovascular risk factor was not considered, since $74 \%$ of the respondents were non-smokers (Abu-Baker et al., 2010).

Based on the BMI, it was found that $24 \%$ of the respondents presented obesity, with a higher prevalence in the ages of 40-49 years, representing $14 \%$ of the obese. And in the study conducted in Spain it represented 22\%.

In comparison with the study carried out in the municipality of Santa Rosa de Osos in Colombia, it was found that the most important cardiovascular risk factors were headed by low physical activity (56.6\%), unlike the present study where this condition was not evaluated as a risk factor of relevance since sport and physical activity are one of the most motivated behaviors in the company (Martin-Espinosa et al., 2017). Mentioning this study conducted in the Antioquian population also resulted in central obesity (52.7\%), dyslipidemia (35.3\%), smoking (19.0\%), metabolic syndrome (19.6\%). and obesity by body mass index (17.4\%) were other cardiovascular risk factors of greater importance. In similarity with our study where central obesity from the abdominal perimeter with a prevalence of $40 \%$ in the ranges of $98-104 \mathrm{~cm}$ (Velez-Alvarez et al., 2015).

Likewise, studies conducted in Colombia in personnel from the administrative area refer to the most prevalent cardiovascular risk factor such as dyslipidemia which is associated as one of the factors that can cause a heart attack after 10 years (Machado-Alba and Machado-Duque, 2013). Therefore, comparing our result, dyslipidemia is one of the most prevalent cardiovascular risk factors.

Regarding the prevalence of hypertension, both studies showed a low prevalence of arterial hypertension, with a measurement of less than $50 \%$ of the surveyed population. The values for each study were, for an oil company, $6 \%$ and for the Antioquia study, $17.1 \%$ (Rabani et al., 2018). A difference to take into account in this risk factor, for our study there was a high prevalence of pre-hypertensive (22\%) with respect to hypertension and the highest percentage was represented by workers without diagnosis of this condition (72\%) while that in the study in Santa Rosa de Oso, the condition of pre-hypertension was not taken into account.

Comparing with the study carried out in Brazil, it was determined that there is a relation in the high prevalence of overweight evidenced in the youngest sector, in the workers evaluated in oil company it was found that this condition represented $56 \%$ of the respondents and the prevalence was in relatively younger ages (30-39 years) with $36 \%$. 


\section{CONCLUSION}

From the results presented, from their discussion and from the background of the literature exposed through the article, the following main conclusions can be obtained.

It was found that the highest prevalence in cardiovascular risk factors associated with workers were obesity and dyslipidemia, these modifiable risk factors being an opportunity to improve the health conditions of workers and a lower prevalence of hypertension, diabetes and smoking.

On the other hand, the Framminghan analysis shows that the population studied has a low overall risk for cardiovascular disease. Regarding the risk assessment for cardiovascular disease at 10 years (Framminghan Wilson model), a low percentage of evaluated patients are likely to suffer a CVD at 10 years.

According to the above, the company must establish monitoring and control and implement strategies to prevent workers who are currently at low risk from changing their condition at high risk and in turn, so that those who do not have a short or medium cardiovascular risk term, maintain their condition.

This study had the following limitations: eating habits, psychosocial aspects (depression, anxiety and stress) were not evaluated. Confirmatory tests were not performed for the diagnosis of diabetes and only the fasting glycemia value was taken into account and the presence or not of the diabetes mellitus antecedent for diagnosis of this condition which could lead to false positives in said diagnosis.

\section{REFERENCES}

Abu-Baker, N.N., L. Haddad and O. Mayyas, 2010. Smoking behavior among coronary heart disease patients in Jordan: A model from a developing country. Int. J. Environ. Res. Publ. Health, 7: 751-764.

Assimes, T.L. and R. Roberts, 2016. Genetics: Implications for prevention and management of coronary artery disease. J. Am. Coll. Cardiol., 68: 2797-2818.

Balcıoglu, A.S. and H. Muderrisoglu, 2015. Diabetes and cardiac autonomic neuropathy: Clinical manifestations, cardiovascular consequences, diagnosis and treatment. World J. Diabetes, 6: 80-91.

Balog, J.E., 2017. The meaning of health in the era of value-based care. Cureus, Vol. 9, No. 2. 10.7759/cureus.1042.

Bhurosy, T. and R. Jeewon, 2014. Overweight and obesity epidemic in developing countries: A problem with diet, physical activity, or socioeconomic status? Scient. World J., Vol. 2014. 10.1155/2014/964236.
Bitton, A. and T. Gaziano, 2010. The framingham heart study's impact on global risk assessment. Prog. Cardiovasc. Dis., 53: 68-78.

Braveman, P. and L. Gottlieb, 2014. The social determinants of health: it's time to consider the causes of the causes. Publ. Health Rep., 129: 19-31.

Chan, R.S.M. and J. Woo, 2010. Prevention of overweight and obesity: how effective is the current public health approach. Int. J. Environ. Res. Public Health, 7: 765-783.

Gómez, E., 2016. [Introduction, epidemiology of heart failure and the history of cardiac failure clinics in Colombia]. Rev. Colom. Cardiol., 23: 6-12.

Hajar, R., 2017. Risk factors for coronary artery disease: Historical perspectives. Heart Views, 18: 109-114.

Juhnke, C., S. Bethge and A.C. Muhlbacher, 2016. A review on methods of risk adjustment and their use in integrated healthcare systems. Int. J. Integr. Care, Vol. 16, No. 4. 10.5334/ijic.2500

Machado-Alba, J.E. and M.E. Machado-Duque, 2013. [Cardiovascular risk factors prevalence among patients with dyslipidemia in Colombia]. Rev. Perú. Med. Exp. Salud Publica, 30: 205-211.

Martin-Espinosa, N., A. Diez-Fernandez, M. Sanchez-Lopez, I. Rivero-Merino and L.L.D. La Cruz et al., 2017. Prevalence of high blood pressure and association with obesity in Spanish schoolchildren aged 4-6 years old. PloS One, Vol. 12. 10.1371/journal.pone.0170926.

Mendis, S., 2005. Cardiovascular risk assessment and management in developing countries. Vasc. Health Risk Manage., 1: 15-18.

Mensah, G.A., G.S. Wei, P.D. Sorlie, L.J. Fine and Y. Rosenberg et al., 2017. Decline in cardiovascular mortality: Possible causes and implications. Circulation Res., 120: 366-380.

Mohammadbeigi, A., E. Moshiri, N. Mohammadsalehi, H. Ansari and A. Ahmadi, 2015. Dyslipidemia prevalence in Iranian adult men: The impact of population-based screening on the detection of undiagnosed patients. World J. Mens Health, 33: 167-173.

Mora-Pabón, G., 2018. [Research on heart failure in Colombia, time to take a step forward]. Rev. Fac. Med., 66: 137-138.

Ofori-Asenso, R. and D. Garcia, 2016. Cardiovascular diseases in Ghana within the context of globalization. Cardiovasc. Diagn. Ther., 6: 67-77.

Pagidipati, N.J. and T.A. Gaziano, 2013. Estimating deaths from cardiovascular disease: A review of global methodologies of mortality measurement. Circulation, 127: 749-756.

Patiño-Villada, F.A., E.F. Arango-Vélez, M.A. QuinteroVelásquez and M.M. Arenas-Sosa, 2011. [Cardiovascular risk factors in an urban Colombia population]. Rev. Salud Pública, 13: 433-445. 
Posada, J., I. Orjuela, I. Ahumada, J. Palacio, L. Vargas and C. Severiche, 2019. Risks associated to blood hypertension in workers of maintenance of a mining complex of carbon of la guajira colombiana. J. Eng. Applied Sci., 14: 615-622.

Rabani, S.H. M. Sardarinia, S. Akbarpour, F. Azizi, D. Khalili and F. Hadaegh, 2018. 12-year trends in cardiovascular risk factors (2002-2005 through 20112014) in patients with cardiovascular diseases: Tehran lipid and glucose study. PLoS One, Vol. 13, No. 5. 10.1371/journal.pone.0195543.

Sanchis-Gomar, F., C. Perez-Quilis, R. Leischik and A. Lucia, 2016. Epidemiology of coronary heart disease and acute coronary syndrome. Ann. Transl. Med., Vol. 4, No. 13. 10.21037/atm.2016.06.33.

Shah, A. and M. Afzal, 2013. Prevalence of diabetes and hypertension and association with various risk factors among different Muslim populations of Manipur, India. J. Diabetes Metab. Disord., Vol. 12, No. 1.

Soler, E.P. and V.C. Ruiz, 2010. Epidemiology and risk factors of cerebral ischemia and ischemic heart diseases: Similarities and differences. Curr. Cardiol. Rev., 6: 138-149.
Thimbleby, H., 2013. Technology and the future of healthcare. J. Public Health Res., 2: 160167.

Upadhyay, R.K., 2015. Emerging risk biomarkers in cardiovascular diseases and disorders. J. Lipids, Vol. 2015. 10.1155/2015/971453.

Vélez-Alvarez, C., L.M. Gil-Obando, C.L. Avila-Rendón and A. López-López, 2015. [Cardiovascular risk factors and variables associated in people aged 20-79 years in Manizales, Colombia]. Univ. Salud, 17: 3246.

Wachter, J.K. and P.L. Yorio, 2014. A system of safety management practices and worker engagement for reducing and preventing accidents: An empirical and theoretical investigation. Accid. Anal. Prev., 68: 117130.

Yusufali, A., N. Bazargani, K. Muhammed, A. Gabroun and A. Al-Mazrooei et al., 2015. Opportunistic screening for CVD risk factors: The Dubai shopping for cardiovascular risk study (DISCOVERY). Global Heart, 10: 265-272. 